\title{
5G Enabled Technologies for Smart Education
}

\author{
Delali Kwasi Dake ${ }^{1}$, Ben Adjei Ofosu ${ }^{2}$ \\ Department of ICT Education \\ University of Education, Winneba \\ Winneba, Ghana
}

\begin{abstract}
G technology use cases depicts the prospects of 5G network model to revolutionize Industry and Education is not an exception. The 5G model in general is made up of three main blocks: Enhanced Mobile Broadband, Massive Machine Type Communication and Ultra Reliable and Low Latency Communication. Within these blocks are the services 5G offers to users. In this paper, we focus on Educational users as beneficiaries of $5 \mathrm{G}$ technologies. The modern day Educational Institutions can benefit from the deployment of 5G-enabled services adapted to this sector. We proposed frameworks relating 5G and its disruptive technologies in advancing tools that will propel the idea of a Smart Educational System. This paper hence provides a comprehensive discussion on $5 \mathrm{G}$ technologies that will facilitate new teaching and learning trends in Educational environment.
\end{abstract}

Keywords-5G Networks; smart education; smart campus; machine learning; artificial intelligence; big data; internet of things

\section{INTRODUCTION}

5G technology stands for 5th Generation of the Mobile Technology (MT). This MT has evolved from the past four decades starting from $1 \mathrm{G}$ (1st Generation), $2 \mathrm{G}$ (2nd Generation), 3G (3rd Generation), 4G (4th Generation) and now the expected $5 \mathrm{G}[1]$. 1G is being used for voice calls, $2 \mathrm{G}$ for SMS which was later improved to 2.5 to help support browsing activities on the internet. $3 \mathrm{G}$ is for mobile television with Global Positioning System (GPS) and video conferencing which are all in use today. $4 \mathrm{G}$ is an improvement on $3 \mathrm{G}$ with extra services for higher data exchange. Long Term Evolution (LTE) is the standard for high speed wireless communication and boosted the abilities of $4 \mathrm{G}$ with a higher downloading and uploading speed rates.

The 5G MT will bring about a "Network Society" where many network technologies including Millimeter Wave (mmWave), Massive Multiple Input Multiple Output (mMiMo), Full Duplex, Cognitive Radio (CR) Technology, ZigBee, Bluetooth Low Energy (BLE) and Dynamic Spectrum Access (DSA) would be integrated to provide improvement in terms of reliability, availability, flexibility, energy efficiency and low latency. These technologies are important requirements for advancements in Internet of Things (IoT) [2]. The integration of these technologies for $5 \mathrm{G}$ also offers support for Augmented and Virtual Reality which are effective technologies applicable to Education. Therefore the imminence arrival and commercialisation of 5G and its technologies must lead to a discussion about its potential benefit to Education.
The rapid integration of Information communication and technology tools (ICTs) in education in aiding synchronous and asynchronous lesson delivery and new ways to access information and knowledge are important in the teaching process. Instructors and Learners mostly adopt the YouTube in accessing educational video contents with most institutions integrating web based educational platforms: the Moodle, Edmodo and Edsby [3] to facilitate school assessments and management. Other smart devices have also emerged in adaptation to intelligent tutoring systems, personalized learning and Recommender Systems [4]. Academic Institutions are yet to fully benefit from the Integration of Internet of Things (IoT) in Education that will completely lead to the idea of Smart Education.

The data generated by these IoT devices and mobile phones on campuses will lead to an era of Big Data that can be analysed to shape and create a dynamic educational system with intelligent gathering concepts. The intelligence gathered can suggest new learning patterns, curriculum re-design and better management decisions on running academic institutions based on facts.

$5 \mathrm{G}$ is going to disrupt the ICT ecosystem thereby having a greater impact on the concept of Smart Education (SmE), Smart University (SmU) and a Smart Campus (SmC). This has exerted pressure on Education to adapt all the necessary technology advancement to attain the level of the new age of industry (Industry 4.0). From various perspectives, $5 \mathrm{G}$ will be a facilitator and a quickening agent of Industry 4.0 and SMART Education (SmE, SmU, SmC).

\section{REVIEW OF LITERATURE}

Author in [5] concentrated on the architecture of the ecosystem of $5 \mathrm{G}$ network. The research addressed the three main categories of the $5 \mathrm{G}$ Ecosystem which are the Enhanced Mobile Broadband (eMBB), Ultra-reliable and Low-latency Communications (uRLLC), and Massive Machine Type Communications (mMTC). It continued to elaborate on the various services within the three blocks of the $5 \mathrm{G}$ Ecosystem. It uncovered other services that seeks to meet the demands of individuals and industries. Due to the lack of recognition for Education in the 5G Ecosystem Model, the researcher proposed a modification of the model to fully concentrate on services that could directly be applied in Education.

Author in [6] focused on the attributes of 5G such as higher data rates, low latency and efficient use of spectrum with coexistence of heterogeneous networks for a successful compatibility of $5 \mathrm{G}$ and Internet of Things. This paper proposed the incorporation of Artificial intelligence as a 
necessity to make competent decisions based on the immense data produced by the activities of users of IoT devices. It addressed the use of Artificial intelligence to help analyze data in other to extract patterns and make sense of it and then prescribe action to the end devices. It also recommended the need for Artificial Intelligence in IoT devices for device selfanalysis.

Author in [7] fixated the research on 5G as a compatibility for wireless technology and how $5 \mathrm{G}$ network seeks to be a foundation for the development of the World Wide Wireless Web (WWWW) and the Dynamic Adboc Wireless network (DAWN). This research work discussed the reason why the world needs $5 \mathrm{G}$ with its use cases for supporting interactive multimedia, internet and other broadband services and have bidirectional accurate traffic statistics.

Author in [8] explained the significance of IoT and why IoT is an integral part of the daily learning and teaching methodologies. The article concluded that the effective use of IoT systems will improve personalised and interactive learning. This article again focused on the importance of IoT in the mobility tracking of students using intelligent cameras. In [8], author also explained the importance of effective educational applications in leveraging on IoT to transform teaching and learning.

Author in [9] fixated on the educational field where critical learning spaces are prepared to utilize Internet of things. A system was proposed that enables and understudies interfaces with physical objects virtually connected with a subject of learning. The result of their experiment proved that their model improves outcomes in teaching and learning.

Author in [10] research paper recommended cloud computing as a solution to cater for the rapid growth of data. The paper discussed the three models of the cloud computing services which defines the paradigm of what users would use technology for. The three main models of the cloud service in this paper explains what each of the provisions from the cloud computing structure provides. The Software as a Service (SaaS) provides the complete application to a cloud end user. This provides an already made and executable software running over the internet. The Platform as a Service (PaaS) is a virtual environment for provisioning and developing cloud applications and the third model which is the Infrastructure as a Service (IaaS) provides an access of a layer to the IT resources services such as data storage resources, computing resources and communications channel pooled under this service. This service makes it possible for users to use all the intelligence of the cloud service which becomes an appropriate infrastructure for Data Analytics.

\section{5G INTEGRATED TECHNOLOGIES IN EDUCATION}

Considering the services expected of $5 \mathrm{G}$, this research adopted a model from the $5 \mathrm{G}$ ecosystem proposed by Huawei Technology Company Limited, with an integrated concept 'Smart Education' that grouped the services provided by $5 \mathrm{G}$ and adapted to Education as shown in Fig. 1.

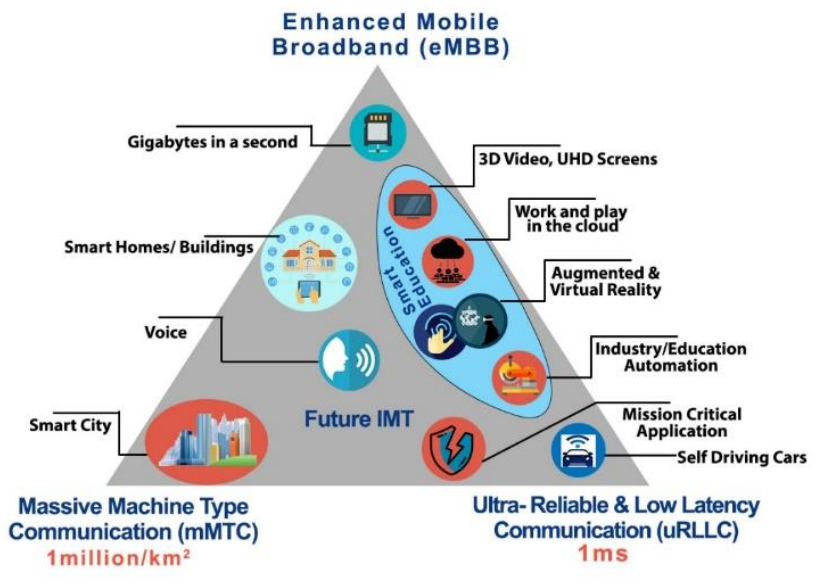

Fig. 1. Smart Education Concept Integrated in Huawei 5G Model.

\section{A. 3D Video, UHD Screens}

$3 \mathrm{D}$ visualization technologies make computer generated images appear more lifelike. This technology has helped in delivering content which are very abstract and difficult to recreate. With 3D virtualization technology, learners sense a greater depth in understanding than just reading. 3D technology led to computer generated images which are equivalent to real world content. This technology has helped integrate simulations and animations in education to better deliver most complicated educational content. Due to the immense benefit of videos in Education, 3D videos adoption to assist in teaching and learning process has become one of the most trending technology to revolutionize Education. Downloading and uploading speed has been a challenge for accessing 3D videos with Fully/Ultra High Definition quality on academic institutions. 5G provides heterogeneous air interfaces to improve spectral efficiency exponentially. 5G provides eMBB data rates for uplink (UL) and downlink (DL) up to $10 \mathrm{~Gb} / \mathrm{s}$ and $20 \mathrm{~Gb} / \mathrm{s}$, respectively. Having such data rate makes it possible for not only downloading and uploading but also, to have a full high definition live video communication with a lower latency. The application of this service in education can assist in synchronous lecture delivering with real time Question and Answer sessions and a live communication between an instructor and a student.

\section{B. Work and Play in the Cloud}

Cloud Computing is a type of Internet-based computing where different services such as server, storage and applications are delivered to an organization's computers and devices through the Internet [11]. 5G services provide the opportunity to work and play in the cloud thus taking advantage of cloud computing technologies with the help of eMBB, uRLLC and mMTC. With this technology, the various departments of an academic institution can take advantage of this provision in reducing the cost of computation, application hosting, content storage and delivery. To be able to fully utilize the services offered by the $5 \mathrm{G}$ Ecosystem, the various models of cloud computing are important. The Ultra Reliable speed with lower latency of $5 \mathrm{G}$ will be the catalyst for the application of this service. Considering the centralised architecture of cloud service providers, there is a prominent delay in accessing 
contents even with a relatively fast internet connectivity. However with $5 \mathrm{G}$, users should be able to use the cloud base models without delays with the aid of Lower latency, higher network availability and ultra-fast data rate exchange. This will keep the responses and executions in real time.

1) Software as a Service (SaaS): As shown in Fig. 2, this model offers a complete application to consumers, as a service on demand. A single instance of the service runs on the cloud and multiple end users are serviced. With the customers (institutions such as UEW), there is no need for upfront investment in software licenses. The SaaS model provides subscription based option online. Currently, SaaS is offered by companies such as Google, Salesforce, Microsoft and Zoho. A service of its kind could be used by Students and Lecturers and can be extended to Departments and Administration.

2) Platform as a Service (PaaS): As shown in Fig. 3, this model offers a layer of software, or development environment that is encapsulated and offered as a service, upon which other higher levels of service can be built. The customer or institution can build applications that will run on the provider's infrastructure. To meet manageability and scalability requirements of the applications, PaaS providers offer a predefined combination of Operating Systems (OS) and application servers that are highly robust and efficient. Students, lecturers and departments related to application and software development can have access to such service without the need to physically install any software on their computers. The PaaS will aid in collaborative projects irrespective of distance.

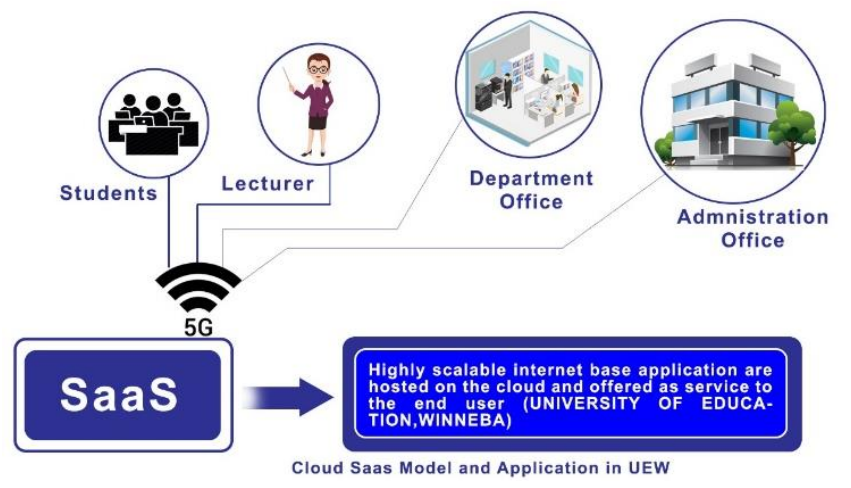

Fig. 2. Cloud SaaS Model and Application in UEW.

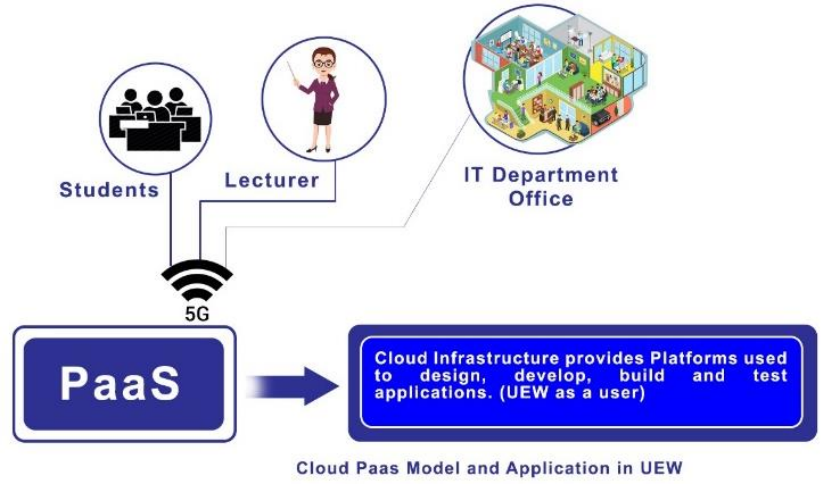

Fig. 3. Cloud PaaS Model and Application in UEW.

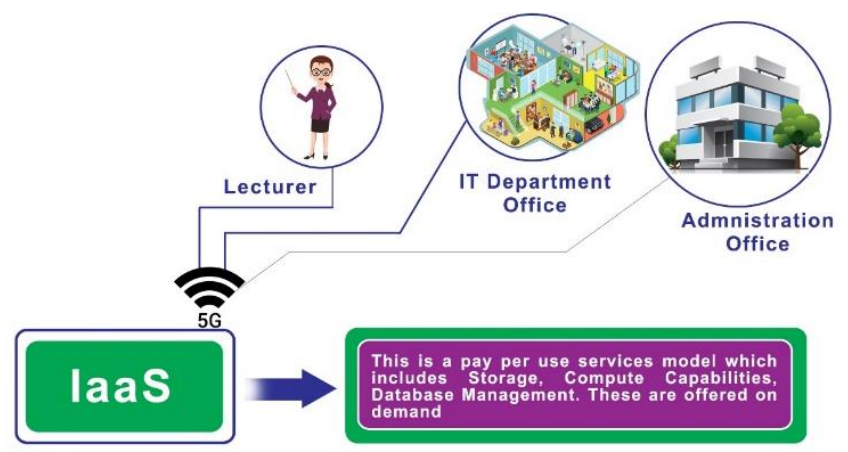

Fig. 4. Cloud IaaS Model and Application in UEW.

3) Infrastructure as a Service (IaaS): As shown in Fig. 4, IaaS provides basic storage and computing capabilities as standardized services over the network. Computer server, storage systems, networking equipments and data centers are pooled and made available to handle workloads. The customer (or institutions such as UEW) would typically deploy their software on the infrastructure.

\section{Augumented and Virtual Reality}

As shown in Fig. 5, the virtual reality is an interactive and haptic computer-generated environment in which users are physically connected through a simulation device to collaboratively execute tasks by perceiving the objects through many senses such as audio-visual, touch and smell senses [12]. Augmented reality is the combination of computer generated and a real content in a constructive view point of the user. Augmented and virtual reality technologies can be combined to achieve a desired objective. The main challenge of these technologies is the available bandwidth, network speed and latency to run it. The solution is offered by $5 \mathrm{G}$ services. These two technologies supported by $5 \mathrm{G}$ can essentially provide enormous benefit to Education in creating smart student, smart instructors and smart administrative team.

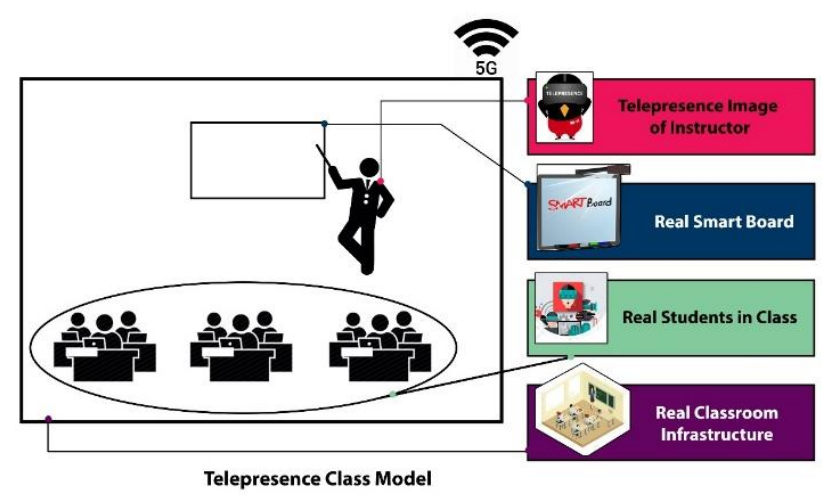

Fig. 5. Augmented and Virtual Reality Applicable to Education.

1) Telepresence Mode (TM): The concept of telepresence could be seen as 'telexistence' where users are enabled by both augmented and virtual visualization to be present at a remote location in a form [13]. The evolution of this concept has led to a technology which is capable of advancing education. Though telepresence with a high end-to-end audio and video conference call was integrated in education, 
somehow much of the precise face-to-face communication such as body language, eye contact, physical presence is still lacking. This face to face communication is one of the reasons that makes traditional classroom interesting. It is therefore important to integrate this immersive communication system in the telepresence concept. Holoportation is an end-to-end system for augmented and virtual reality where users are enabled by both augmented and virtual visualization to be present at a remote location exactly as they are not in any other form [14]. This system is skilled to capture people, objects and motions in full 360 degrees within a constructive room using group of custom depth cameras and then transmitted to a remote participants over a network in real time. The transmission of this communication generate a very large amount of data which makes it challenging to keep communication happening in real time. One factor that needs to be present before holographic communications can wind up completely is an impressive increment in network capacity. The presence of 5G with eMBB and uRLLC will solve this problem. 5G will enable full utilization of this technology. As shown Fig. 5, students in physical classroom are to wear AR or VR Head Mounted Display (HMD) such as Hololens or HTC Vive to see their holoported instructor. The instructor being an agent of knowledge is enabled to continue the transfer of knowledge regardless of the proximity. Students enrolled in Distance Learning modules can use this technology at the comfort of their homes.

\section{2) Full and Hybrid Virtual Model:}

a) Full Virtual Class Model: Full Virtual Class Model (FVCM) is a class where the delivering of learning content, teaching materials and evaluations are fully implemented using virtual and augmented content [15]. Section A in Fig. 6 depicts students accessing augmented and virtual content at the comfort of their desired location. Here, students access contents through the use of electronic devices and Head Mounted Display (HMD). Students accessing such virtual contents would have an in-depth instruction of the topic being delivered. Using the case of Haptic Augmented Technology and Tactile internet, this application can be modified for students to experience the texture of object on Mars. Teaching about Mars by Geography Department at UEW can make good use of such application to help student have a full understanding of this planet which is a futurist travel. Full Virtual Class Model could be adopted by UEW to help strengthen the provision of the Distance Education Program. FVCM doesn't restrict students to their location thereby making it easy for students to have access to class regardless of their location. FVCM can be implemented to help distance education students experience a replication of the traditional classroom.

b) Hybrid Virtual Class Model: Hybrid Virtual Class Model (HVCM) is a class where the delivering of contents and evaluations are accomplished using both traditional classroom and augmented and virtual content. Fig. 6, section B represents a regular class (Traditional Classroom) where there is an instructor and students using HMD. Content of abstract being delivered using the VR content to explain the reality of a concept. The instructor will serve as a guide in directing students on the visuals and help leaners with questions and answers. Example is using the Boulevard application for museum excursions.

\section{Industry / Educational Automation}

5G networks will have a Software-Defined Networking (SDN) architecture that comprises of the control and data planes. Most of the control plane intelligence will instruct data plane to drive the infrastructure [16] [17]. Fig. 7 depicts a scenario of an automated 5G Education setting to enable an intelligent university campus. The emergence of $5 \mathrm{G}$ will provide communication and automation within the classroom and administration offices where Educational Internet of Things (EIoT) and Industrial Internet of Things (IoT) are being used. Educational application "A" represent administration offices with cloud sensor devices and computerized system to perform computation. Educational application " $\mathrm{B}$ " is a classroom with Educational IoT wireless devices such as Smartboard 7000 Series IQ with image detective sensor, sensored door with actuator, sensored chair, smart table with embedded touch screen computer, room temperature sensor, motion and facial expression cameras.

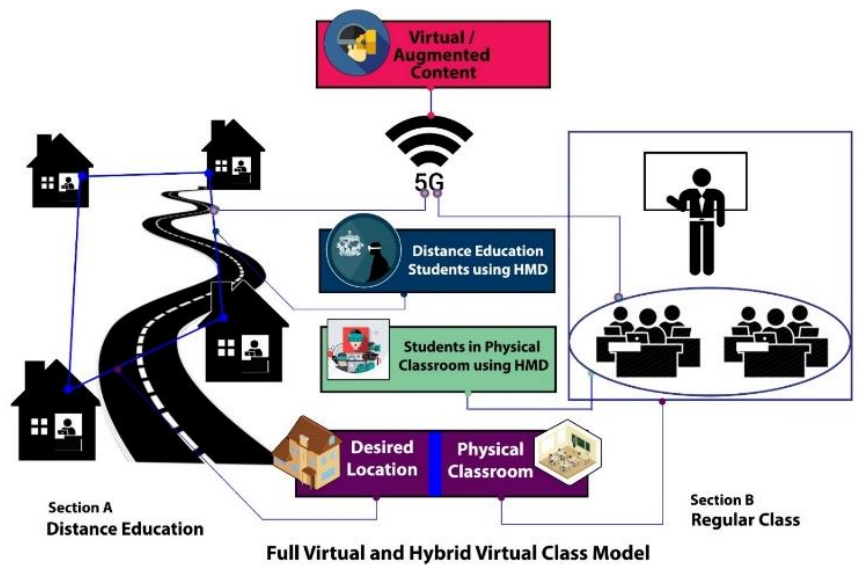

Fig. 6. Full Virtual and Hybrid Class Model.

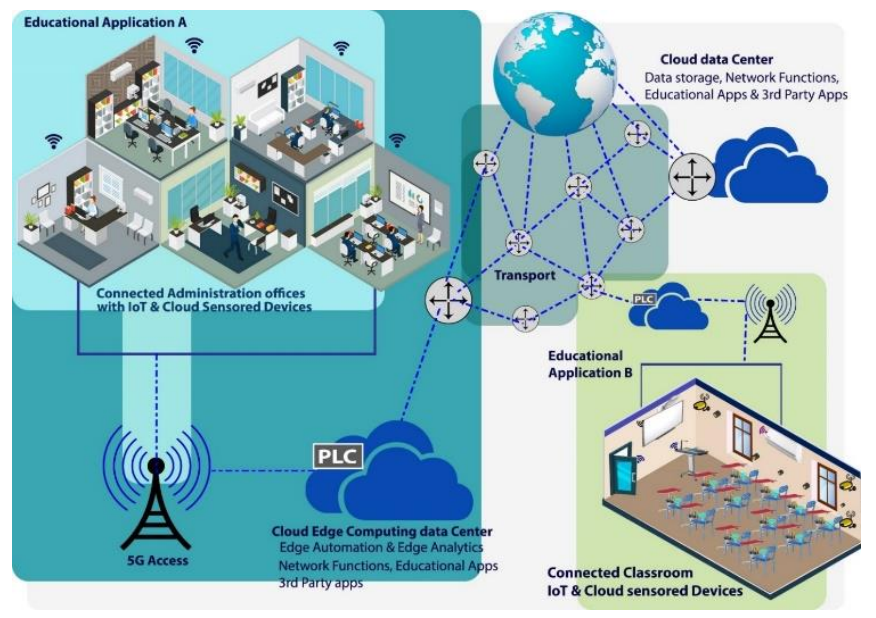

Fig. 7. Prospect Application Scenario of 5G-Enabled Automation in Education. 
Existing automation in Education is highly focused on systems that promote personalised learning through the use of E-Learning system such as the Massive Open Online Courses (MOOCs) [18] and Adaptive Intelligent Tutoring Systems (AITSs) [19]. MOOCs enables flexibility in learning new skills with progress tracking. AITS incorporate built-in expert systems to monitor students' performance and to personalise instructions based on adjustment to students' learning style, current knowledge level and suitable teaching procedures. In addition, Adaptive Hypermedia system is another educational use case which adapts what the student is offered depending on a model of the learner's objectives, proclivity and knowledge. Automation in education over the years has improved learner understanding. These systems are educational interventions to automate education in a different way and improve teaching and learning. However, as far as the prospect of automation in education by $5 \mathrm{G}$ is concerned, machines, devices and humans are to communicate and interact to achieve educational goals. Machines are expected to be intelligent enough to make decisions without the intervention of humans.

Research shows that the revolution in industry (industry 4.0) is pressuring education with demands to keep pace with industry evolution. Therefore it would be very appropriate to adopt the core driving force of industry revolution (automation) and apply it in education. The prospect of automation from $5 \mathrm{G}$ in education takes a different trend from the existing automation in Education. The automation in $5 \mathrm{G}$ seeks to consume the three models of the 5G Ecosystem: eMBB, uRLLC and mMTC thereby utilizing the characteristics of automation, smart interconnection, real-time monitoring and collaborative control. These characteristics enable fast machine learning (ML), machine to machine/man technology (M2M) and Device to Device (D2D) technology.

1) The Role of $M L, M 2 M, D 2 D$ in Automation: Machinelearning (ML) framework involves capturing and maintaining up a rich organised data in order to convert it into a structured information base for various use cases. This enables computers or instructing machines to learn from every past data set and settle on intelligent choices in making best decisions. Machine learning in education helps to personalised learning and instructions. Deployment of this technology interpret patterns and human interaction to support deeper learning and provide users with fast and accurate data in education. M2M is direct connection and communication that can exist between machine to machine, man to machine, machine to man, and machine to mobile network [20]. D2D represent a method of direct communication between two peer nodes [21]. With ML, M2M and D2D, communication between machines, machines and man, and device to device can have an accurate transfer of information to ensure perfect prescription, description and making the best decisions. These technologies work base on established procedures from the obtained data to make decisions and then issues control commands to actuators. Regardless of M2M or D2D, the core problem is the data rate transmission which $5 \mathrm{G}$ will provide.

2) $M L, M 2 M$ and D2D FOR Automation in Education: With reference to Fig. 7, an example of the application of ML,
$\mathrm{M} 2 \mathrm{M}$ and D2D automation can be cited. In Application "B" there is a door with QR code reader to read student's ID card or programmable wearable device. Communication is established between the sensor reader and the actuator to get the door opened. Upon the credentials read from the door's QR code reader, a feed is sent to the Smartboard 7000 Series IQ to power on. The Instructor stores lesson materials on the cloud. With the help of date and time the smartboard automatically makes the e-learning materials available to the instructor. Students on the other hand would have their smart tables turned on using the feed from the sensor in the chairs. Students' smart tables automatically access lesson materials from the cloud. There is room temperature sensor monitoring the temperature of the class. Air-conditioner get powered on and regulated by the feeds from the room temperature sensors. This communication between these machines provide data that is then stored in the cloud for analytics. With data analytics instructors and students can be monitored and report sent to the appropriate office machines at the administration office in Educational application "A". With the eMBB offered by $5 \mathrm{G}$ network model, large amount of data is transmitted in every second whiles the mMTC keeps all the IoT sensors connected. The uRLLC keeps all devices connected with consistent data exchange.

\section{E. Tactile Internet, Over $5 \mathrm{~g}$}

The Tactile Internet increases the capacity to human-tomachine communication by empowering tactile and haptic sensations, and improves the interactions of machines. Tactile internet is a holistic network or grid of networks for remotely getting to, seeing, manipulating, and controlling genuine or virtual objects [22]. This is a sure way for students to have an interactive and haptic feel of Augmented and Virtual reality contents respectively. A typical example is a learner having an aquatic augmented reality excursion. Students can feel the exact temperature of the water body, have a haptic feeling of aquatic plant and animals using Haptic Augmented Reality Technology. For a student to experience the sensational feelings of touching a Wale, it would be an expensive and a highly risky attempt to get a Wale in a real world that would be reachable for a touch, however with the emergence of Tactile Internet, students can have the sense of touching a wale in a virtual classroom. To achieve these interactive haptic feedback in real time, the speed involved in data exchange as well as the latency should meet the exact natural reactions time. Hence a network with Lower Latency is needed. The $5 \mathrm{G}$ Ecosystem can provide the possibility in deploying the Tactile Internet Technology.

\section{CONCLUSION}

The realization of $5 \mathrm{G}$ networks will revolutionize not only industry but will propel a change in Academic Institutions. In this paper, we proposed frameworks and discussed the possibility of using the $5 \mathrm{G}$ Ecosystem and adapting its relevant technologies to Education. This paper discussed the transformation 5G Networks will bring especially in strengthening the teaching and learning process. From this research, the possible deployment 5G technologies in 
Education and the emergence of IoT devices on campuses will serve as a turning point in students learning process, instructors' research and content delivery mechanisms.

\section{REFERENCES}

[1] Meraj, M., \& Kumar, S. (2015). Evolution of mobile wireless technology from 0G to 5G. International Journal of Computer Science and Information Technologies, 6(3), 2545-2551.

[2] N. Javaid, A. Sher, H. Nasir and N. Guizani, "Intelligence in IoT-Based 5G Networks: Opportunities and Challenges," in IEEE Communications Magazine, vol. 56, no. 10, pp. 94-100, OCTOBER 2018. doi: 10.1109/MCOM.2018.1800036.

[3] Gros, B. (2016). The design of smart educational environments. Smart Learning Environments, 3(1), 15.

[4] Klašnja-Milićević, A., Vesin, B., Ivanović, M., Budimac, Z., \& Jain, L. C. (2016). E-learning systems: intelligent techniques for personalization (Vol. 112). Springer.

[5] Demestichas, P., Georgakopoulos, A., Karvounas, D., Tsagkaris, K., Stavroulaki, V., Lu, J., ... \& Yao, J. (2013). 5G on the horizon: Key challenges for the radio-access network. IEEE vehicular technology magazine, 8(3), 47-53.

[6] Javaid, N., Sher, A., Nasir, H., \& Guizani, N. (2018). Intelligence in IoT-Based 5G Networks: Opportunities and Challenges. IEEE Communications Magazine, 56(10), 94-100.

[7] Patil, G. R., \& Wankhade, P. S. (2012). 5G Wireless Technology. world, $1(1)$.

[8] Lee, I. (2017). Big data: Dimensions, evolution, impacts, and challenges. Business Horizons, 60(3), 293-303.

[9] Gómez, J., Huete, J. F., Hoyos, O., Perez, L., \& Grigori, D. (2013). Interaction system based on internet of things as support for education. Procedia Computer Science, 21, 132-139.

[10] Sharma, M. M., Husain, S., \& Ali, M. S. (2017). Cloud computing risks and recommendations for security. International Journal of Latest Research in Science and Technology, 6(1), 52-56.

[11] Li, X., Jiang, W., Jiang, Y., \& Zou, Q. (2012, June). Hadoop applications in bioinformatics. In 2012 7th Open Cirrus Summit (pp. 4852). IEEE.

[12] Bower, M., Howe, C., McCredie, N., Robinson, A., \& Grover, D. (2014). Augmented Reality in education-cases, places and potentials. Educational Media International, 51(1), 1-15.
[13] Kaber, D. B., Riley, J. M., Zhou, R., \& Draper, J. (2000, July). Effects of visual interface design, and control mode and latency on performance, telepresence and workload in a teleoperation task. In Proceedings of the human factors and ergonomics society annual meeting (Vol. 44, No. 5, pp. 503-506). Sage CA: Los Angeles, CA: SAGE Publications.

[14] Orts-Escolano, S., Rhemann, C., Fanello, S., Chang, W., Kowdle, A., Degtyarev, Y., \& Tankovich, V. (2016, October). Holoportation: Virtual $3 d$ teleportation in real-time. In Proceedings of the 29th Annual Symposium on User Interface Software and Technology (pp. 741-754). ACM.

[15] Chiu, H. P., Kaelbling, L. P., \& Lozano-Pérez, T. (2007). Virtual training for multi-view object class recognition. In 2007 IEEE Conference on Computer Vision and Pattern Recognition (pp. 1-8). IEEE.

[16] Kim, H., \& Feamster, N. (2013). Improving network management with software defined networking. IEEE Communications Magazine, 51(2), 114-119.

[17] Shin, M. K., Nam, K. H., \& Kim, H. J. (2012, October). Softwaredefined networking (SDN): A reference architecture and open APIs. In 2012 International Conference on ICT Convergence (ICTC) (pp. 360361). IEEE.

[18] Kizilcec, R. F., Piech, C., \& Schneider, E. (2013, April). Deconstructing disengagement: analyzing learner subpopulations in massive open online courses. In Proceedings of the third international conference on learning analytics and knowledge (pp. 170-179). ACM.

[19] Phobun, P., \& Vicheanpanya, J. (2010). Adaptive intelligent tutoring systems for e-learning systems. Procedia-Social and Behavioral Sciences, 2(2), 4064-4069.

[20] Wu, G., Talwar, S., Johnsson, K., Himayat, N., \& Johnson, K. D. (2011). M2M: From mobile to embedded internet. IEEE Communications Magazine, 49(4), 36-43.

[21] Hakola, S., Chen, T., Lehtomaki, J., \& Koskela, T. (2010, April). Device-to-device (D2D) communication in cellular networkperformance analysis of optimum and practical communication mode selection. In 2010 IEEE wireless communication and networking conference (pp. 1-6). IEEE.

[22] Cheng, J., Chen, W., Tao, F., \& Lin, C. L. (2018). Industrial IoT in 5G environment towards smart manufacturing. Journal of Industrial Information Integration, 10, 10-19. 